\title{
The Logarithmic Spiral: Mathematical Aspects and Modeling in Turbulence
}

\author{
Haris J. Catrakis \\ Mechanical and Aerospace Engineering, University of California, Irvine \\ 4200 Engineering Gateway, Irvine, CA 92697, USA
}

Tel: 1-949-824-4028 E-mail: catrakis@uci.edu

Received: March 2, 2011 Accepted: March 21, 2011 doi:10.5539/jmr.v3n3p3

This research is supported by the National Science Foundation Career Award

\begin{abstract}
We consider mathematical aspects of the logarithmic spiral and its utility in turbulence modeling. We consider mathematically the set of point crossings resulting from a linear intersection through the center of a logarithmic spiral. We derive analytically the fractal dimension as a function of scale for this set of crossings. We also derive analytically the power spectrum of the thresholded function corresponding to these point crossings. Our mathematical results have implications for turbulence modeling which are motivated by experimental observations of logarithmic spiral structures of scalar fields in turbulence.
\end{abstract}

Keywords: Spirals, Level Crossings, Fractal Dimensions, Spectra, Turbulence

\section{Introduction}

Spirals are frequently observed and utilized in a variety of phenomena including galaxies, biological organisms, as well as turbulent flows (Catrakis 2000, Aldridge 1998, Fonseca 1993, Moffatt 1993, Gilbert 1988, Pickover 1988, Burton 1973, Montomery 1970, Francis 1940, Castle 1934, Jennings 1901). In turbulence, logarithmic spirals have been observed for scalar fields (Everson \& Sreenivasan 1992) and algebraic spirals have been observed for vortex fields (Lundgren 1982, Flohr \& Vassilicos 1992, Vassilicos \& Brasseur 1996, Angilella \& Vassilicos 1999). Whereas algebraic spirals have been studied extensively before (Gilbert 1988, Moffatt 1993, Vassilicos \& Brasseur 1996), we will focus on logarithmic spirals whose fractal and spectral aspects have not been studied in detail before to our knowledge. The logarithmic spiral, which is traditionally called a logarithmic spiral even though it has an exponential function for the radial coordinate $r$ as a function of the angle $\theta$, is expressed as $r=b e^{-a \theta}$ where $a$ is any non-zero constant and $b$ is any positive constant. It is called historically the logarithmic spiral in terms of the logarithmic dependence of the angle $\theta$ as a function of the radial coordinate $r$, i.e. $\theta=-\ln (r / b) / a$. It is also sometimes called the equiangular spiral. The algebraic spiral is expressed as $r=b \theta^{-a}$ and the Archimedes spiral $r=b \theta$ is a special case of the algebraic spiral. While properties of importance to turbulence studies such as the fractal dimension and power spectrum are known for the algebraic spiral (Gilbert 1988, Moffatt 1993, Vassilicos \& Brasseur 1996), such properties have not been previously derived to our knowledge for the logarithmic spiral and our work focuses therefore on the properties of the logarithmic spiral.

In this paper, we consider analytically the set of point crossings in a linear intersection of the logarithmic spiral through its center. In section 2 , we analytically explore the crossing scales, i.e. the spacing scales between successive point crossings, in order to obtain the probability density function of crossing scales. This enables us to derive the fractal dimension as a function of scale for the point crossings of the logarithmic spiral. In section 3, we derive the power spectrum of the thresholded function corresponding to the point crossings. We discuss the implications of our results for turbulence modeling. We summarize in section 4 our conclusions.

\section{The Logarithmic Spiral: Derivation of the Fractal Dimension as a Function of Scale}

The logarithmic spiral can be expressed in polar coordinates, or equivalently in other forms such as Cartesian coordinates or parametric coordinates, as follows:

$$
\begin{aligned}
r & =b e^{-a \theta} \\
x^{2}+y^{2} & =b^{2} e^{-2 a \tan ^{-1}(y / x)} \\
x & =b e^{-a \theta} \cos \theta \\
y & =b e^{-a \theta} \sin \theta
\end{aligned}
$$

where we will choose $\theta \geq 0, a>0$ to ensure that the spiral converges to the origin as $\theta \rightarrow \infty$, and $b>0$. An example of the logarithmic spiral is shown in figure 1.

If we intersect the spiral through its center along the horizontal axis, the intersections of the spiral will occur at angles 
given by $\theta=0,2 \pi, 4 \pi, 6 \pi, \ldots$ for $x>0$ and $\theta=\pi, 3 \pi, 5 \pi, 7 \pi, \ldots$ for $x<0$ as can be seen in figure 1 .

Thus, the angles of the intersections of the spiral with the horizontal axis are as follows:

$$
\theta_{k}=k \pi
$$

where $k=0, \ldots, N$ and in the limit we let $N \rightarrow \infty$. The corresponding horizontal locations of these crossing points are therefore:

$$
x_{k}=(-1)^{k} b e^{-a \theta_{k}}=(-1)^{k} b e^{-a k \pi}
$$

and thus in terms of their absolute values these are:

$$
\left|x_{k}\right|=b e^{-a \theta_{k}}
$$

Therefore, the crossing scales $l_{k}$ which are the spacings between successive crossing points can be expressed as:

$$
l_{k}=\left|x_{k}-x_{k+2}\right|=(-1)^{k}\left(x_{k}-x_{k+2}\right)=b\left(e^{-a k \pi}-e^{-a(k+2) \pi}\right)
$$

where the reason the indices are $k$ and $k+2$ is because every pair of successive crossing points occurs on either the right or the left side of the spiral as in figure 1. We show in figure 2 a schematic of the crossing scales $l_{k}$ and the corresponding pairs of successive crossing points along the intersection through the center of the spiral.

The probability density function (pdf) of the crossing scales can be written therefore as a sum of delta functions because all of the crossing scales in equation 5 have distinct values. Thus, the pdf of crossing scales can be expressed as:

$$
p(l)=\frac{1}{N} \sum_{k=0}^{N-1} \delta\left(l-l_{k}\right)
$$

for finite $N$, with the understanding that the analysis of the crossing scales for the entire logarithmic spiral correspond to the limit $N \rightarrow \infty$.

We can now use the following general relation derived by the author (Catrakis 2000) which relates directly the pdf $p(l)$ of crossing scales to the fractal dimension $D(\lambda)$ as a function of the coverage scale $\lambda$, i.e. the partition scale corresponding to successively partitioning the entire interval containing all the point crossings, as follows:

$$
D(\lambda)=1-\frac{\lambda \int_{\lambda}^{\infty} p(l) d l}{\int_{0}^{\lambda} \int_{\lambda^{\prime}}^{\infty} p(l) d l d \lambda^{\prime}}
$$

Substituting from equation 6 for the pdf $p(l)$ of crossing scales of the logarithmic spiral, we obtain the following result for the fractal dimension $D(\lambda)$ which we express for clarity this way:

$$
D(\lambda)=1-\frac{\lambda A(\lambda)}{B(\lambda)}
$$

where the functions $A(\lambda)$ and $B(\lambda)$ are given respectively by:

$$
A(\lambda)=\int_{\lambda}^{\infty} p(l) d l=\left\{\begin{array}{cc}
0, & \lambda>l_{0} \\
\frac{k}{N}, & l_{k-1}>\lambda>l_{k} \quad, \quad k=1,2, \ldots, N-1 \\
1, & \lambda<l_{N-1}
\end{array}\right.
$$

and, 


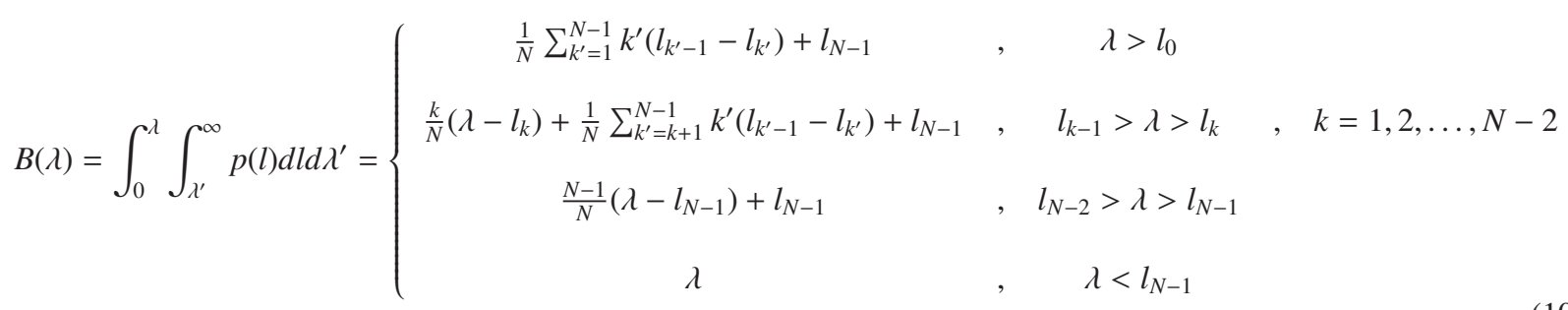

We plot the functions $A(\lambda)$ and $B(\lambda)$ in figures 3,4 , and 5 .

Our full analytical result for the dimension $D(\lambda)$ follows therefore by combining equations 8,9 , and 10 , so that we obtain the following expression for the fractal dimension $D(\lambda)$ as a function of scale $\lambda$ for the entire range of scales:

$$
D(\lambda)=\int_{0}^{\lambda} \int_{\lambda^{\prime}}^{\infty} p(l) d l d \lambda^{\prime}=\left\{\begin{array}{cc}
1 & \lambda>l_{0} \\
1-\frac{\lambda \frac{k}{N}}{\frac{k}{N}\left(\lambda-l_{k}\right)+\frac{1}{N} \sum_{k^{\prime}=k+1}^{N-1} k^{\prime}\left(l_{k^{\prime}-1}-l_{k^{\prime}}\right)+l_{N-1}} & , \quad l_{k-1}>\lambda>l_{k} \quad, k=1,2, \ldots, N-2 \\
1-\frac{N\left(\frac{N-1}{N}\right)}{\frac{(N-1)}{N}\left(\lambda-l_{N-1}\right)+l_{N-1}} & , \quad l_{N-2}>\lambda>l_{N-1} \\
0 & , \quad \lambda<l_{N-1}
\end{array}\right.
$$

We plot the dimension in figure 6 where we see that there is an overall increase in the dimension with increasing scale, i.e. there is no constant dimension, and moreover there is non-monotonicity associated with the consecutive transitions between successive parts of the spiral at its intersection with the horizontal axis as can be seen in figure 1 . This is a useful result in the context of turbulence because there are various observations such as experimental studies by several authors cited in the work of Catrakis (2000) which show an overall increase of the dimension with increasing scale. The logarithmic spiral thus offers one possible model for such observed behavior and it is a particularly plausible model because there are direct experimental observations of logarithmic spirals found in scalar fields in turbulence (Everson \& Sreenivasan 1992).

\section{The Logarithmic Spiral: Derivation of the Power Spectrum}

In this section, we derive analytically the power spectrum for a thresholded function corresponding to the logarithmic spiral. As shown in figure 7, we have constructed a thresholded function $f(x)$ as a summation series of step functions such that the thresholded function has steps at successive pairs of point crossings of the logarithmic spiral through its center along the horizontal axis. Thus, we write analytically the thresholded function $f(x)$ as:

$$
f(x)=\sum_{k=0}^{\infty}\left[\left[H\left(x-x_{4 k+2}\right)-H\left(x-x_{4 k}\right)\right]+\left[H\left(x-x_{4 k+1}\right)-H\left(x-x_{4 k+3}\right)\right]\right]
$$

where $H$ is the Heaviside unit step function.

In equation 12, the first pair of Heaviside functions corresponds to pairs of crossings at positive locations and the second pair of Heaviside functions corresponds to pairs of crossings at negative locations, on the horizontal axis, as is evident in figure 7 .

We proceed to express the thresholded function $f(x)$ as follows:

$$
f(x)=\sum_{k=0}^{\infty}\left[W_{x_{4 k}-x_{4 k+2}}\left(x-x_{4 k+2}\right)+W_{x_{4 k+3}-x_{4 k+1}}\left(x-x_{4 k+1}\right)\right]
$$

where $W$ is the finite-width step function defined in the present analysis as follows: 


$$
W_{a}(x-b)=H(x-b)-H(x-b-a)=\left\{\begin{array}{cc}
0, & x<b \\
1 & , \quad b \leq x<b+a \\
0 & , \quad x \geq b+a
\end{array}\right.
$$

where $a>0$ and thus $W$ represents a finite-width unit step function which has the finite width $a$. The finite-width step function $W$ is helpful for taking Fourier transforms analytically.

Thus, the reason that we express the thresholded function $f(x)$ in equation 13 in terms of the finite-width step function $W$ from equation 14 is because this will help us to take analytically the Fourier transform of the thresholded function $f(x)$ in terms of a linear superposition of the individual Fourier transforms of the finite-width step functions.

We can now analytically take the Fourier transform of the thresholded function from equation 13 because the Fourier transform $\hat{W}_{a, b}(\omega)$ of the finite-width step function $W_{a}(x-b)$ is given by:

$$
\hat{W}_{a, b}(\omega)=\frac{1}{\sqrt{2 \pi}} \int_{b}^{b+a} e^{-i \omega x} d x=\frac{1}{\sqrt{2 \pi}} \frac{e^{-i \omega b}-e^{-i \omega(b+a)}}{i \omega}=\sqrt{\frac{2}{\pi}} e^{-i \omega\left(b+\frac{a}{2}\right)} \frac{\sin (\omega a / 2)}{\omega}
$$

Therefore, the Fourier transform of the thresholded function from equations 13 and 14 is:

$$
\begin{aligned}
& \hat{f}(\omega)=\sqrt{\frac{2}{\pi}} \sum_{k=0}^{\infty}\left[e^{-i \frac{x_{4 k}+x_{4 k+2}}{2} \omega} \frac{\sin \left(\frac{x_{4 k}-x_{4 k+2}}{2} \omega\right)}{\omega}+e^{-i \frac{x_{4 k+3}+x_{4 k+1}}{2} \omega} \frac{\sin \left(\frac{x_{4 k+3}-x_{4 k+1}}{2} \omega\right)}{\omega}\right] \\
& =\sqrt{\frac{2}{\pi}} \sum_{k=0}^{\infty}\left[(-1)^{-4 k} e^{-i \frac{x_{4 k}+x_{4 k+2}}{2} \omega} \frac{\sin \left(\frac{l_{4 k}}{2} \omega\right)}{\omega}-(-1)^{-(4 k+1)} e^{-i \frac{x_{4 k+3}+x_{4 k+1}}{2} \omega} \frac{\sin \left(\frac{l_{4 k+1}}{2} \omega\right)}{\omega}\right] \\
& =\sqrt{\frac{2}{\pi}} \sum_{k=0}^{\infty}\left[e^{-i \frac{x_{4 k}+x_{4 k+2}}{2} \omega} \frac{\sin \left(\frac{l_{4 k}}{2} \omega\right)}{\omega}+e^{-i \frac{x_{4 k+3}+x_{4 k+1}}{2} \omega} \frac{\sin \left(\frac{l_{4 k+1}}{2} \omega\right)}{\omega}\right]
\end{aligned}
$$

where we have used $l_{4 k}=(-1)^{4 k}\left(x_{4 k}-x_{4 k+2}\right)=x_{4 k}-x_{4 k+2}$ and $l_{4 k+1}=(-1)^{4 k+1}\left(x_{4 k+1}-x_{4 k+3}\right)=x_{4 k+3}-x_{4 k+1}$ from equation 5.

Thus, we can now analytically derive the power spectral density of our thresholded function by taking the square of the modulus of the Fourier transform of the signal, i.e. by taking the product of the Fourier transform and its complex conjugate. The power spectrum can be obtained therefore analytically as:

$$
\begin{aligned}
& |\hat{f}(\omega)|^{2}=\hat{f}(\omega) \hat{f}^{*}(\omega) \\
& =\sqrt{\frac{2}{\pi}} \sum_{k=0}^{\infty}\left[e^{-i \frac{x_{4 k}+x_{4 k+2}}{2} \omega} \frac{\sin \left(\frac{l_{4 k}}{2} \omega\right)}{\omega}+e^{-i \frac{x_{4 k+3}+x_{4 k+1}}{2} \omega} \frac{\sin \left(\frac{l_{4 k+1}}{2} \omega\right)}{\omega}\right] \\
& \times \sqrt{\frac{2}{\pi}} \sum_{k^{\prime}=0}^{\infty}\left[e^{i \frac{x_{4 k^{\prime}}+x_{4 k^{\prime}+2}}{2} \omega} \frac{\sin \left(\frac{l_{4 k^{\prime}}}{2} \omega\right)}{\omega}+e^{i \frac{x_{4 k^{\prime}+3}+x_{4 k^{\prime}+1}}{2} \omega} \frac{\sin \left(\frac{l_{4 k^{\prime}+1}}{2} \omega\right)}{\omega}\right]
\end{aligned}
$$

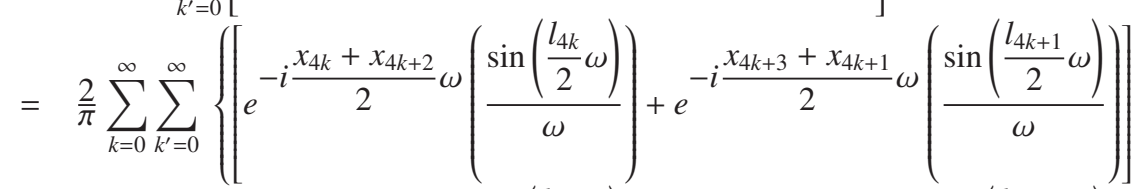

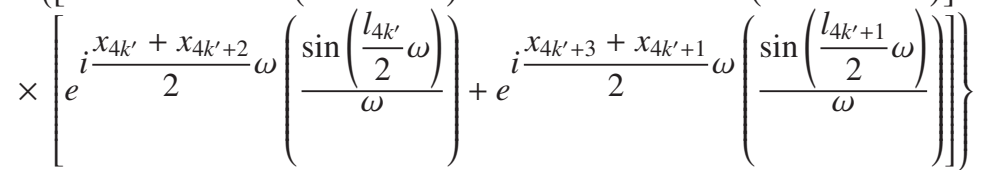

We show in figure 8 the power spectrum for the thresholded function corresponding to the point crossings of the logarithmic spiral. A parabolic fit in log-log coordinates is also shown as a dashed curve and gives a good fit at high frequencies. The parabola is of interest because a parabolic behavior would mean that the power spectrum is approximately lognormal.

Our analysis of the logarithmic spiral agrees with observations of scalar fields in turbulence. There are empirical observations and measurements of scalar fluctuations in turbulence (e.g. Everson \& Sreenivasan 1992, Miller \& Dimotakis 1996) which indicate a lognormal power spectrum in linear-linear coordinates, i.e. a parabolic power spectrum in log-log coordinates. Thus, our present analysis offers a theoretical explanation of such experimental findings and suggests that logarithmic spirals have an important role as physical structures for mathematical modeling of scalar fields in turbulence. 


\section{Conclusions}

We have presented an analysis of mathematical properties of the logarithmic spiral. An important motivation in turbulence for studying logarithmic spirals is that there are experimental observations of logarithmic spiral structures of scalar fields in turbulence. By considering mathematically the set of point crossings resulting from a linear intersection through the center of a logarithmic spiral, we derived analytically the fractal dimension as a function of scale for this set of crossings. We also derived analytically the power spectrum of the thresholded function corresponding to these point crossings. In the context of turbulence, these results are useful because they provide a mathematical model that can be utilized in studies of the behavior of scalar fields in turbulent flows.

\section{Acknowledgment}

The author acknowledges helpful and useful comments by the Editor and by the Reviewers.

\section{References}

Aldridge, A. E. (1998). Brachiopod outline and the importance of the logarithmic spiral. Paleobiology, 24, $215-226$.

Angilella, J. R. \& Vassilicos, J. C. (1999). Time-dependent geometry and energy distribution in a spiral vortex layer. Phys. Rev. E, 59, 5427-5439, doi:10.1103/PhysRevE.59.5427, http://dx.doi.org/10.1103/PhysRevE.59.5427

Burton, W. B. (1973). The kinematics of galactic spiral structure. Pub. Astron. Soc. Pacific, 85, 679-703, doi:10.1086/129529, http://dx.doi.org/10.1086/129529

Castle, E. S. (1934). The spiral growth of single cells. Science, 80, 362-363, doi:10.1126/science.80.2077.362, http://dx.doi.org/10.1126/science.80.2077.362

Catrakis, H. J. (2000). Distribution of scales in turbulence. Phys. Rev. E, 62, 564-578, doi:10.1103/PhysRevE.62.564, http://dx.doi.org/10.1103/PhysRevE.62.564

Everson, R. M. \& Sreenivasan, K. R. (1992). Accumulation rates of spiral-like structures in fluid flows. Proc. R. Soc. Lond. A, 437, 391-401, doi:10.1098/rspa.1992.0068, http://dx.doi.org/10.1098/rspa.1992.0068

Flohr, P. \& Vassilicos, J. C. (1997). Accelerated scalar dissipation in a vortex. J. Fluid Mech., 348, 295-317, doi:10.1017/S0022112097006927, http://dx.doi.org/10.1017/S0022112097006927

Fonseca, R. (1993). Shape and order in organic nature: The nautilus pompilius. Leonardo, 26, 201-204, doi:10.2307/1575811, http://dx.doi.org/10.2307/1575811

Francis, W. D. (1940). Spiral systems in the organization of living material. Plant Phys., 15, 301-309, doi:10.1104/pp.15.2.301, http://dx.doi.org/10.1104/pp.15.2.301

Fuchikami, N. \& Ishioka, S. (2004). Statistics of level crossing intervals. Proc. SPIE, 5471, 29-37, doi:10.1117/12.544594, http://dx.doi.org/10.1117/12.544594

Gilbert, A. D. (1988). Spiral structures and spectra in two-dimensional turbulence. J. Fluid Mech., 193, 475-497, doi:10.1017/S0022112088002228, http://dx.doi.org/10.1017/S0022112088002228

Jennings, H. S. (1901). On the significance of the spiral swimming of organisms. Amer. Nat., 35, 369-378, doi:10.1086/277922, http://dx.doi.org/10.1086/277922

Lundgren, T. S. (1982). Strained spiral vortex model for turbulent fine structure. Phys. Fluids, 25, 2193-2203, doi:10.1063/1.863957, http://dx.doi.org/10.1063/1.863957

Miller, P. L. \& Dimotakis, P. E. (1996). Measurements of scalar power spectra in high Schmidt number turbulent jets. $J$. Fluid Mech., 308, 129-146, doi:10.1017/S0022112096001425, http://dx.doi.org/10.1017/S0022112096001425

Moffatt, H. K. (1993). Spiral structures in turbulent flow. In M. Farge, J. C. R. Hunt, \& J. C. Vassilicos (Eds.), Wavelets, fractals, and fourier transforms (pp. 121-129). New York: Clarendon Press.

Pickover, C. A. (1988). Mathematics and beauty: A sampling of spirals and 'strange' spirals in science, nature, and art. Leonardo, 21, 173-181, doi:10.2307/1578555, http://dx.doi.org/10.2307/1578555

Montgomery, W. M. (1970). The origins of the spiral theory of phyllotaxis. J. Hist. Bio., 3, 299-323, doi:10.1007/BF00137356, http://dx.doi.org/10.1007/BF00137356

Vassilicos, J. C. \& Brasseur, J. G. (1996). Self-similar spiral flow structure in low Reynolds number isotropic and decaying turbulence. Phys. Rev. E, 54, 467-485, doi:10.1103/PhysRevE.54.467, http://dx.doi.org/10.1103/PhysRevE.54.467 


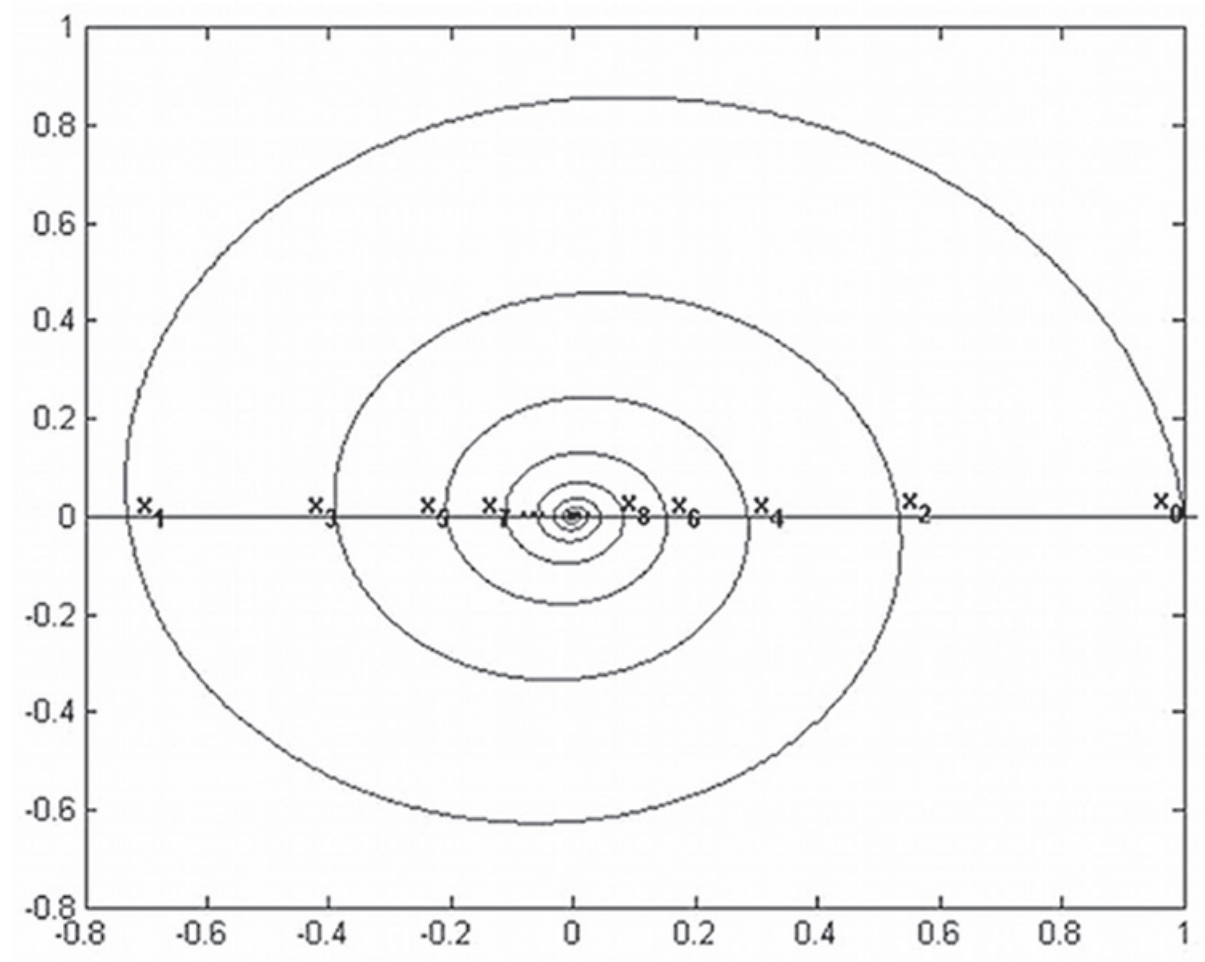

Figure 1. Example of a logarithmic spiral and the set of crossing points formed by the intersections of the spiral through its center along the horizontal axis. Successive crossings are labeled as $x_{k}$ with $k=0, \ldots, N$, where in the limit $N \rightarrow \infty$

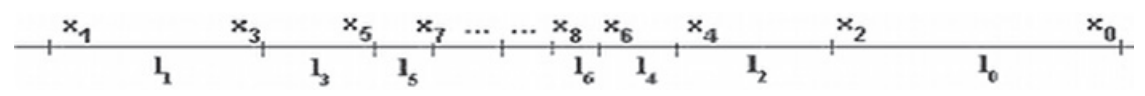

Figure 2. Schematic of the crossing scales $l_{k}$, i.e. the spacings between pairs of successive crossing points $x_{k}$ and $x_{k+2}$, along the intersection through the center of the logarithmic spiral

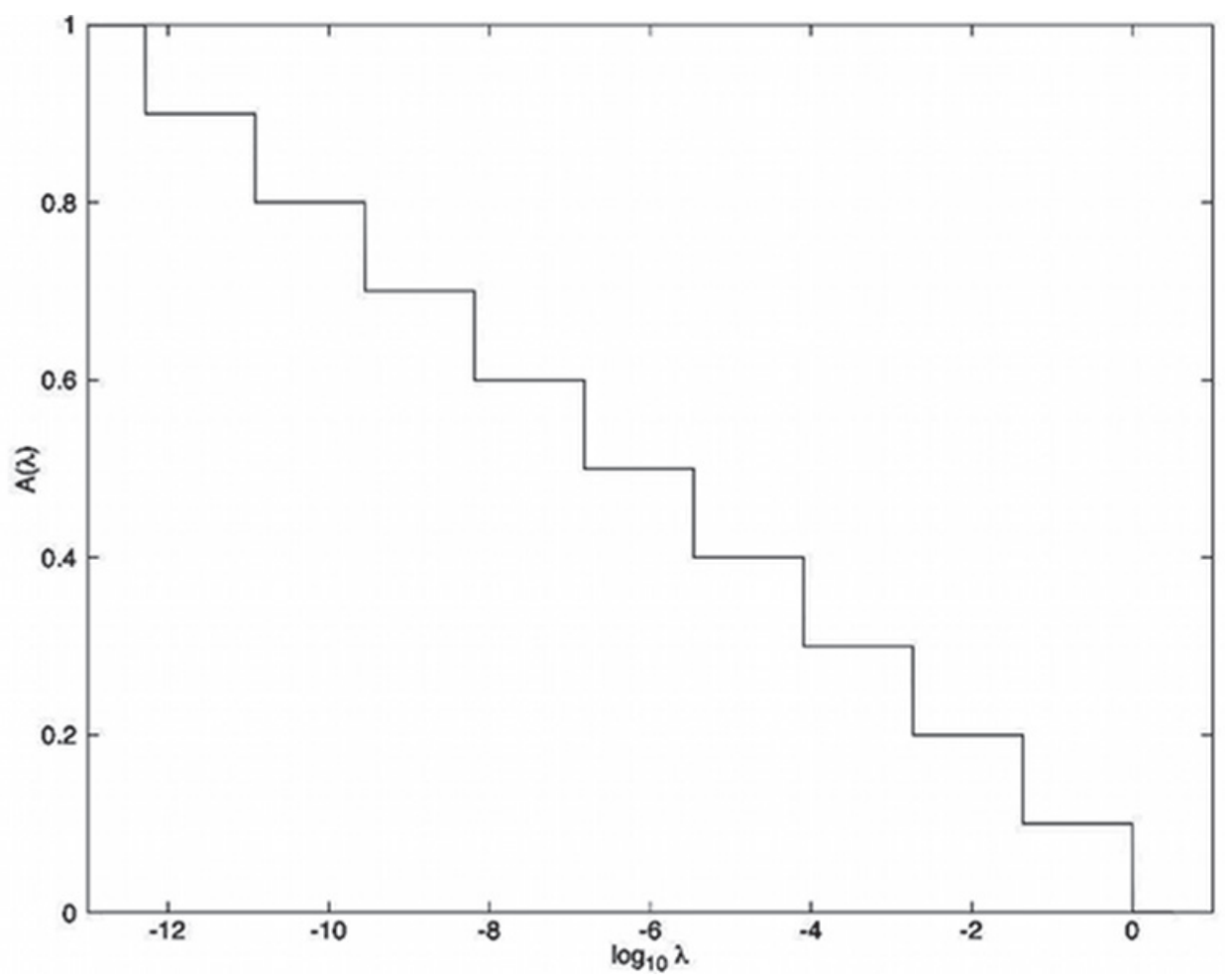

Figure 3. Plot of the function $A(\lambda)$ from equation 9 showing its dependence on logarithmic scale 


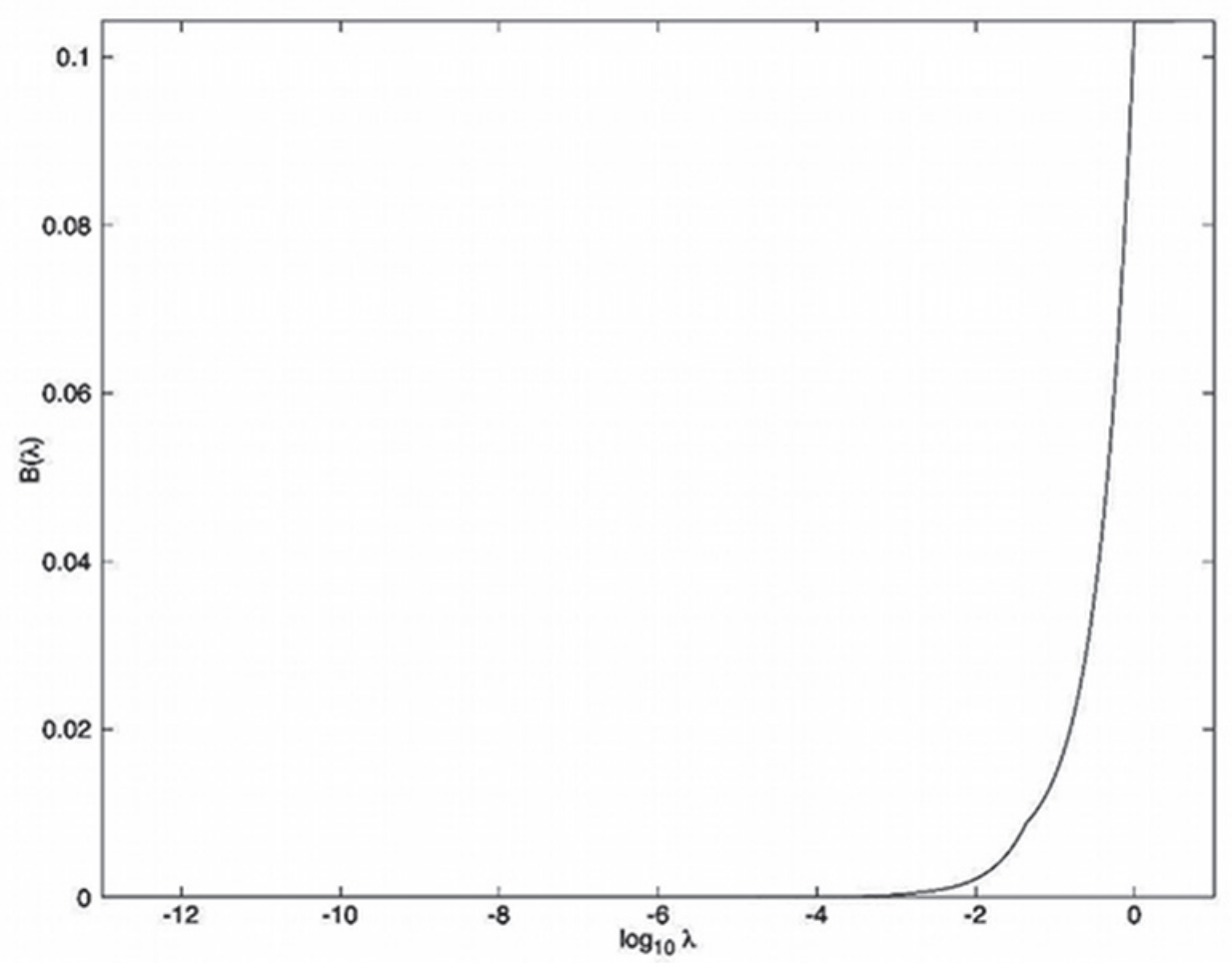

Figure 4. Plot of the function $B(\lambda)$ from equation 10 showing its dependence on logarithmic scale

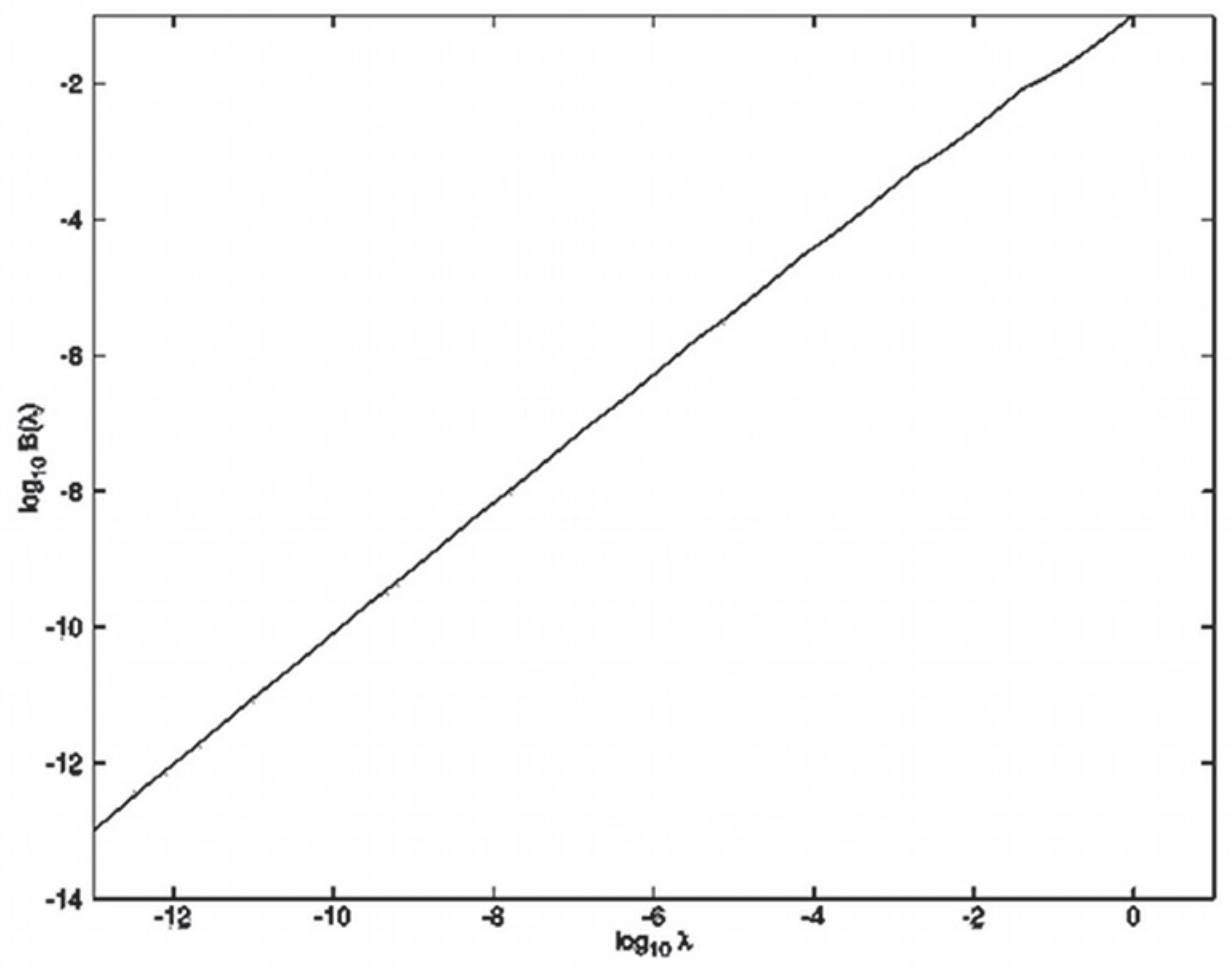

Figure 5. Plot of the logarithm of the function $B(\lambda)$ from equation 10 showing its dependence on logarithmic scale 


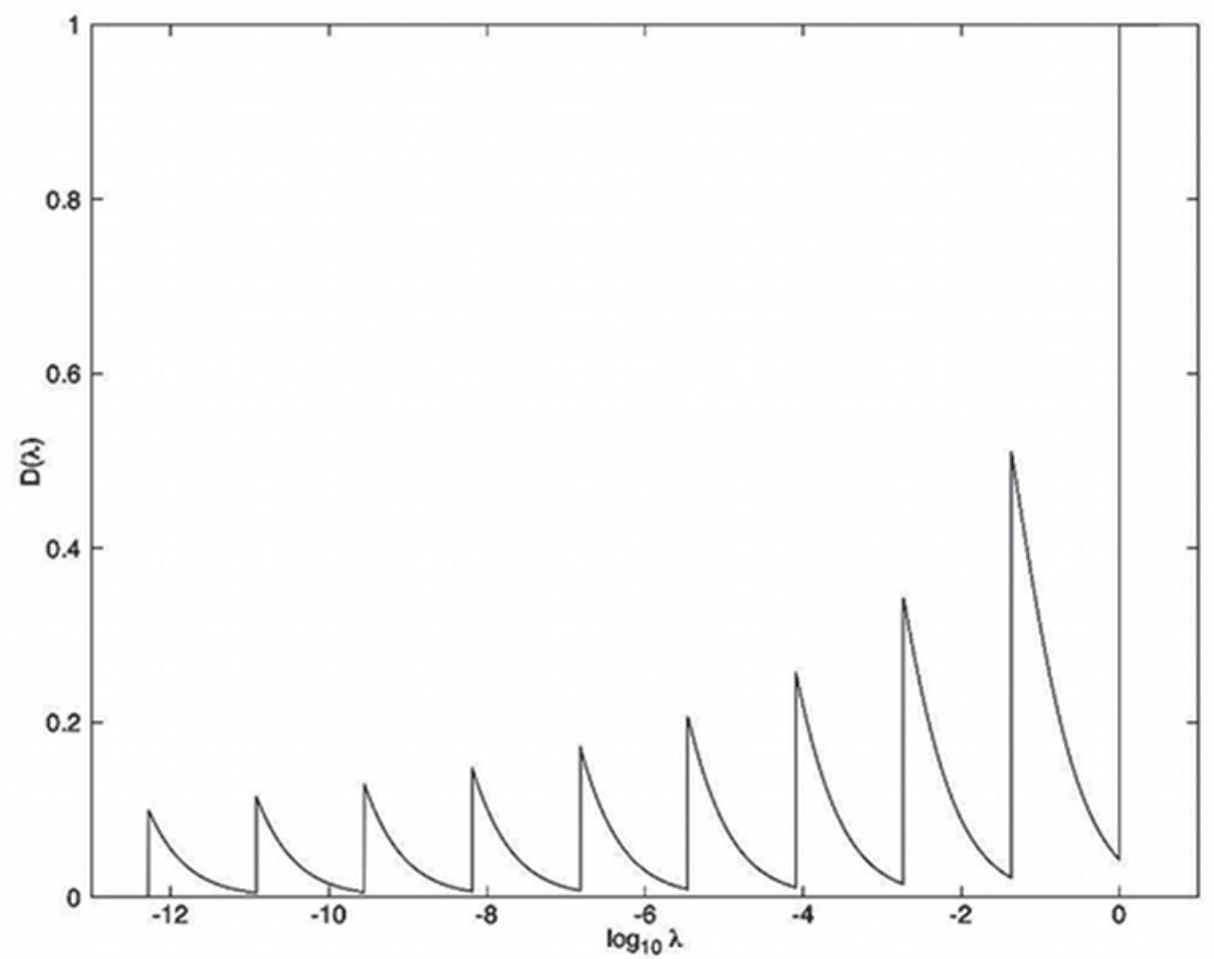

Figure 6. The dimension $D(\lambda)$ of the logarithmic spiral plotted as a function of logarithmic scale

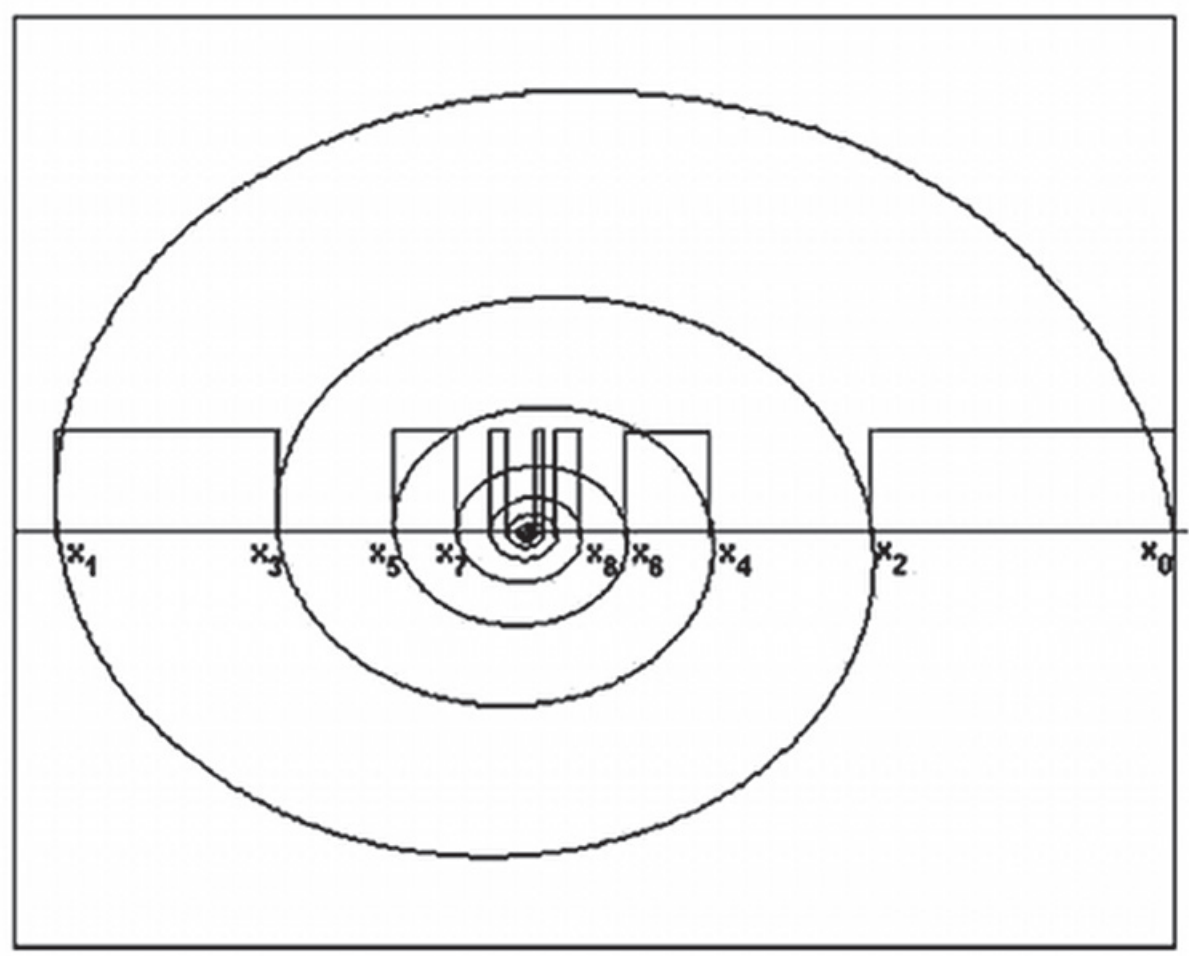

Figure 7. Thresholded function created in terms of step functions between successive pairs of point crossings of the logarithmic spiral through its center along the horizontal axis 


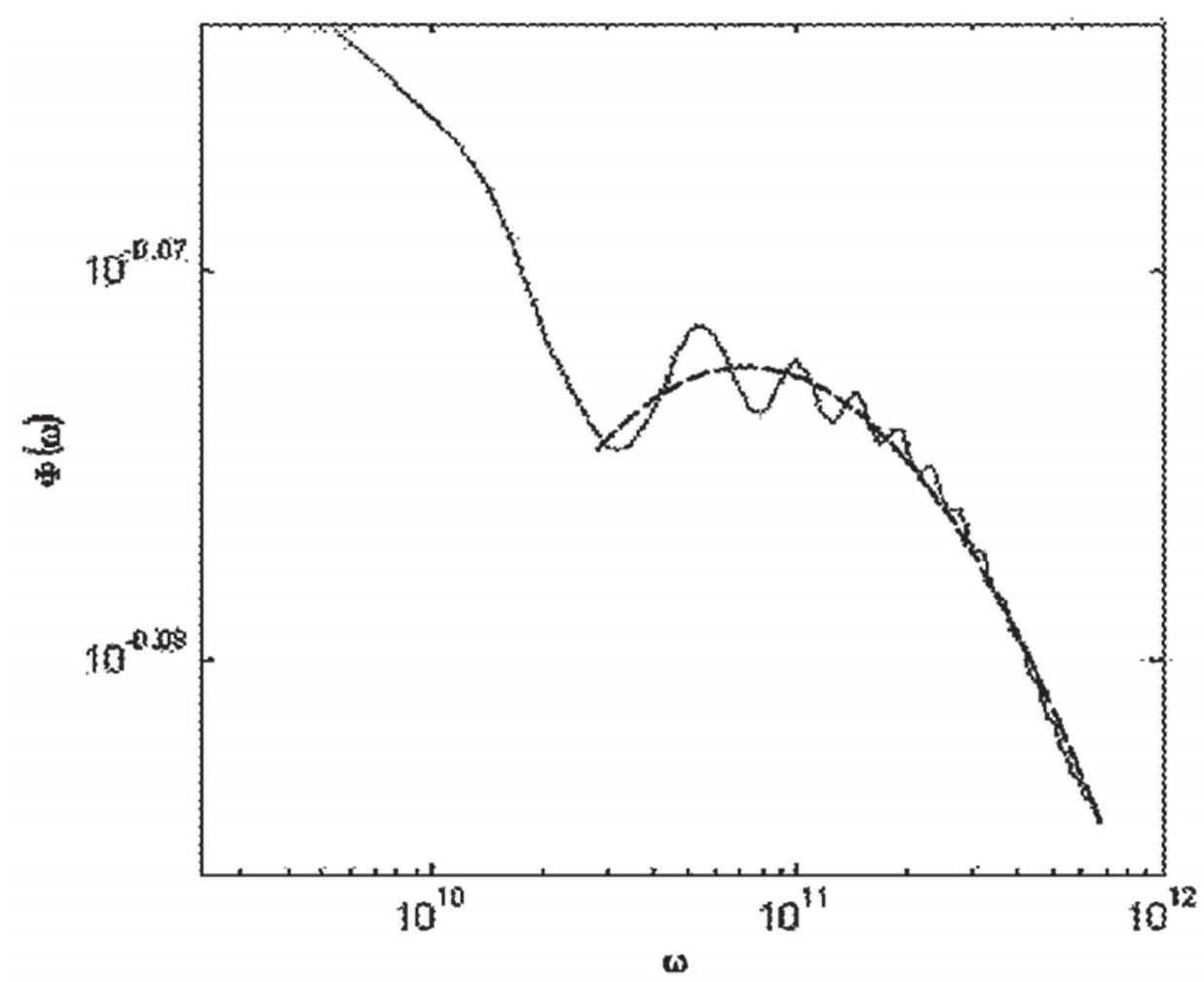

Figure 8. Log-log plot of the analytical power spectrum (solid curve) and comparison to a parabola (dashed curve) which corresponds to the lognormal power spectrum model 